\title{
TEORIA DE UTILIDADE EPISTÊMICA E O ARGUMENTO DA ACURÁCIA A FAVOR DO PROBABILISMO
}

\author{
Epistemic Utility Theory and the Accuracy Argument for Probabilism
}

Resumo: Diferente dos argumentos tradicionais na literatura em epistemologia formal, o argumento da acurácia gradacional de James Joyce fornece uma justificação epistêmica do probabilismo. $\mathrm{O}$ seu argumento pretende mostrar que funções probabilísticas de graus de crença têm maior grau de acurácia, ou menor grau de inacurácia, do que qualquer outra função não probabilística de crença gradual. Neste artigo, reconstruímos e examinamos a sua proposta, que é definida como uma estratégia de utilidade epistêmica. Também fornecemos uma aplicação de uma determinada medida de inacurácia (Brier score) sobre diferentes funções de crença gradual. Por fim, discutimos alguns problemas que foram levantados contra a versão original do argumento de Joyce e concluímos com algumas sugestões de resposta a esses problemas.

Palavras-chave: Acurácia, Bayesianismo, Graus de Crença, Probabilismo, Utilidade Epistêmica.

\begin{abstract}
Unlike the traditional arguments in the formal epistemology literature, James Joyce's gradational accuracy argument provides an epistemic vindication of probabilism. Joyce's argument aims to show that probabilistic credences (or probabilistic degrees of belief) are more accurate, or less inaccurate, than any other non-probabilistic credences. In this paper, we reconstruct and evaluate Joyce's proposal, which is considered an epistemic utility approach. We also provide an application of an inaccuracy measure (Brier score) to different credence functions. Lastly, we discuss some problems that have been raised against the original version of Joyce's argument, and we conclude by suggesting some responses to these problems.
\end{abstract}

Keywords: Accuracy, Bayesianism, Degrees of Belief, Probabilism, Epistemic Utility.

* Doutorando em Filosofia pela PUCRS, bolsista CAPES. E-mail: al.neiva@ gmail.com.

\begin{tabular}{|c|c|c|c|c|c|}
\hline intuitio & $\begin{array}{c}\text { ISSN } \\
1983-4012\end{array}$ & Porto Alegre & Vol.10 $-\mathrm{N}^{\mathrm{o}} .1$ & $\begin{array}{l}\text { Julho } \\
2017\end{array}$ & p.15-24 \\
\hline
\end{tabular}




\section{Preliminares}

Bayesianos consideram crença como um fenômeno gradual. Nesse sentido, agentes doxásticos podem ter uma diversidade de graus de crença (credences) sobre diferentes proposições. Trata-se de um modelo quantitativo de crença: um agente pode atribuir qualquer valor real $\varepsilon$ de uma gama de valores entre 0 e 1 a uma proposição, ou seja, $\{\varepsilon \in \mathbb{R}: 0 \leq \varepsilon \leq 1\}$. Assim, crer com grau máximo em uma proposição $p$ corresponde à atitude de certeza absoluta na sua verdade, ao passo que crer com grau mínimo em uma proposição $q$ corresponde à atitude de certeza absoluta na sua falsidade e, igualmente, valores intermediários de tal intervalo representam diferentes graus intermediários de crença. Portanto, graus 0 e 1 de crença representam casos de graus extremos de crença.

Neste artigo, nosso foco está concentrado em uma restrição sobre o modelo de graus de crença. ${ }^{1}$ Mais particularmente, o propósito deste artigo é reconstruir e examinar um tipo de argumento a favor de uma norma de racionalidade para graus de crença, uma norma conhecida como probabilismo, que constitui parte importante do programa Bayesiano em epistemologia formal. ${ }^{2}$ Essa norma impõe uma restrição racional sincrônica sobre o modelo de crença gradual: os graus de crença de um agente em um dado tempo devem ser probabilidades. Existem tipos diferentes de argumentos a favor do probabilismo. A maioria deles, no entanto, oferece uma justificação pragmática em defesa do probabilismo. O Dutch book sincrônico impõe que graus de crença devem corresponder a quocientes e comportamentos de aposta e mostra que o agente terá perda monetária se ele viola os axiomas de probabilidade; o argumento baseado no teorema representacional recorre à noção de preferências, sendo que estas devem obedecer a um determinado conjunto de restrições de racionalidade pragmática; e o argumento da calibragem afirma que probabilidades subjetivas, ou crenças graduais, devem ser calibradas, isto é, devem corresponder a frequências relativas sobre estados do mundo. ${ }^{3}$ Nenhum deles fornece uma defesa propriamente epistêmica do probabilismo.

\footnotetext{
${ }^{1}$ Não vamos avaliar como a noção de crença gradual se relaciona com a noção de crença tradicional em epistemologia, o modelo de crença simpliciter ou modelo qualitativo, onde crença é considerada uma atitude de tudoou-nada, a saber, não admite graus. A esse respeito, ver David Christensen (2004, cap. 2) e Hannes Leitgeb (2013). O primeiro oferece um bom resumo sobre tentativas de unificar os dois modelos de crença e o segundo propõe uma tese atraente na qual crença simpliciter é reduzida a graus de crença, defendendo que as propriedades e restrições lógicas de ambos modelos são preservadas.

${ }^{2}$ Existem outras normas e restrições de racionalidade probabilística que não serão tratadas aqui, mas são igualmente fundamentais para o Bayesianismo. Para citar algumas, o princípio de condicionalização estrita exige que uma função $c r_{\text {new }}(h)$ deve ser igual a $c r_{\text {old }}(h \mid e)$ quando $e$ é aprendida pela experiência por um agente (onde $\left.c r_{\text {new }}(e)=1\right)$. O princípio de indiferença diz que um agente deve distribuir os seus graus de crença, ou probabilidades subjetivas, de modo simétrico em situações onde ele não tem evidência a favor de alguma hipótese de uma partição. Sobre justificações dos princípios de condicionalização e de indiferença em termos de acurácia, ver respectivamente Hilary Greaves \& David Wallace (2006) e Richard Pettigrew (2014b).

${ }^{3}$ Defensores do Dutch book sincrônico incluem F. P. Ramsey (1950 [1926]) e de Finetti (1964 [1937]), o argumento baseado no teorema representacional é sustentado por Patrick Maher (1993 e 1997) e entre os proponentes do
}

\begin{tabular}{|c|c|c|c|c|c|}
\hline intuitio & $\begin{array}{c}\text { ISSN } \\
1983-4012\end{array}$ & Porto Alegre & Vol.10 - No.1 & $\begin{array}{l}\text { Julho } \\
2017\end{array}$ & p.15-24 \\
\hline
\end{tabular}


Entretanto, nos últimos anos surgiram propostas de justificação do probabilismo em termos de metas estritamente epistêmicas. Nessa perspectiva, a defesa oferecida por James Joyce (1998 e 2009) é considerada original e bastante promissora para o empreendimento do projeto Bayesiano. $\mathrm{O}$ seu argumento está baseado na noção de acurácia gradacional, ${ }^{4}$ que é considerada, conforme Richard Pettigrew (2013, 2015 e 2016), um tipo de medida de utilidade epistêmica. ${ }^{5}$ Em linhas gerais, quanto maior é o grau de acurácia (ou menor é o grau de inacurácia) de um conjunto de graus de crença, melhor ele representa o estado do mundo: mais próximo ele está da verdade. Quanto menor é o seu grau de acurácia (ou maior é o seu grau de inacurácia), pior ele representa o estado do mundo: mais distante ele está da verdade. Desse modo, Joyce pretende demonstrar que funções probabilísticas de graus de crença, aquelas que satisfazem o cálculo de probabilidades padrão (axiomas de Kolmogorov), são mais acuradas do que qualquer outra função alternativa não probabilística de graus de crença. Vamos nos restringir à primeira versão do argumento de Joyce (1998).

Este artigo está organizado da seguinte maneira. Na seção 2, definiremos propriamente o que é o probabilismo e trataremos de alguns aspectos técnicos relacionados ao cálculo de probabilidades. Na seção 3, apresentaremos brevemente alguns aspectos centrais da teoria de utilidade epistêmica. O argumento de Joyce (1998) pode ser classificado como um tipo de argumento de utilidade epistêmica a favor do probabilismo. Em seguida, na seção 4, vamos nos ocupar mais detidamente do argumento da acurácia gradacional e de seus aspectos formais. Uma discussão sobre três dos principais problemas levantados contra o argumento original de Joyce constitui o objeto da seção 5. Por último, apresentaremos algumas indicações de resposta e prospectos de pesquisa em teoria de utilidade epistêmica.

\section{Probabilismo}

Graus de crença representam a força ou o grau de confiança de um agente $S$ sobre um conjunto de proposições, ou seja, o quão fortemente $S$ toma certas proposições que ele considera ou entretém mentalmente como verdadeiras. Se $S$ crê que $p$ com grau $\chi$ e crê que $q$ com grau $\varepsilon$ tal que $\chi<\varepsilon$, então o grau de confiança de $S$ em $q$ é maior do que o seu grau em $p$ ou, simplesmente, $S$ crê mais fortemente em $q$ do que $p$. Pelo bem da simplicidade, assumiremos que agentes têm unicamente valores definidos ou

argumento da calibragem estão van Fraassen (1983) e Marc Lange (1999). Para uma avaliação crítica de tais argumentos, ver Alan Hájek (2009).

${ }^{4}$ Preferimos o termo acurácia e seus correlatos ao termo precisão para tradução de accuracy. Com isso, evitamos ambiguidades e confusões desse conceito com outra distinção igualmente importante entre graus precisos de crença e graus imprecisos de crença.

${ }^{5}$ Um argumento precursor a favor do probabilismo tendo como base a noção de acurácia pode ser encontrado na proposta desenvolvida por Roger Rosenkrantz (1981).

\begin{tabular}{|c|c|l|l|l|l|}
\hline intuitio & $\begin{array}{c}\text { ISSN } \\
1983-4012\end{array}$ & Porto Alegre & Vol.10 $-\mathrm{N}^{\circ} .1$ & $\begin{array}{l}\text { Julho } \\
2017\end{array}$ & p.15-24 \\
\hline
\end{tabular}


precisos de graus de crença. Isso envolve uma certa quantidade de idealização, pois não reflete muitas das nossas crenças mundanas das quais só temos um grau de crença impreciso ou aproximado. ${ }^{6}$

Nesta altura, podemos definir o probabilismo nos seguintes termos:

$\langle\mathbf{P R O B}\rangle$ : Graus de crença de um agente racional $S$ devem satisfazer os axiomas de probabilidade.

Significa que qualquer função de graus de $\operatorname{crença~} \operatorname{cr}(\bullet)$ de um agente racional $S$ deve ser uma função de probabilidade em qualquer tempo $t$. Uma função $\operatorname{cr}(\bullet)$ não é necessariamente probabilística. Mas se ela está de acordo com PROB, então naturalmente ela obedece aos axiomas de probabilidade. Precisamos, no entanto, definir exatamente qual é a versão do cálculo de probabilidades que está sendo assumida em PROB.

Para tanto, seguiremos um conjunto de axiomas de probabilidade bastante fraco tal que probabilidades categóricas ou incondicionais são primitivas. ${ }^{7}$ Em primeiro lugar, $S$ tem graus de crença sobre uma álgebra de proposições $\mathcal{F}$. A função de graus de crença de $S$ está, portanto, restringida a $\mathcal{F}$. Por conseguinte, $\operatorname{cr}(\bullet)$ toma cada $p_{i} \in \mathcal{F}$ e associa um número real a $p_{i}$, uma quantidade que corresponde ao grau de crença de $S$ em $p_{i}$. Tal conjunto ou álgebra de proposições $\mathcal{F}$ está sob negação e combinações contáveis de conjunção e disjunção, ou seja, assumiremos que ela forma uma $\sigma$-álgebra sobre um conjunto $\Omega$. Normalmente, define-se $\mathcal{F}$ sobre um conjunto não-vazio de possibilidades ou espaço de amostra $\Omega$. Quando $\operatorname{cr}(\bullet)$ é probabilística, temos um espaço de probabilidades $\langle\Omega, \mathcal{F}, c r\rangle$. Supondo que $c r: \mathcal{F} \rightarrow \mathbb{R}$ e para qualquer $p_{i} \in \mathcal{F}$, temos os seguintes axiomas de probabilidade:

(1) $\operatorname{cr}\left(p_{i}\right) \geq 0$;

(2a) $\operatorname{cr}(\top)=1$;

(2b) $\operatorname{cr}(\perp)=0$;

(3) Se $\left\{p_{1}, \ldots, p_{n}\right\}$ é um conjunto ${ }^{8}$ de proposições mutuamente exclusivas ou incompatíveis, então $\operatorname{cr}\left(p_{1} \vee \ldots \vee p_{n}\right)=\operatorname{cr}\left(p_{1}\right)+\ldots+\operatorname{cr}\left(p_{n}\right)^{9}$

\footnotetext{
${ }^{6} \mathrm{Um}$ grau de crença $\varepsilon$ em uma proposição qualquer é impreciso ou indeterminado quando $\varepsilon$ corresponde a um intervalo $[\mathrm{a}, \mathrm{b}] \subseteq[0,1]$, ou seja, $\varepsilon$ diz respeito a todo subconjunto $[\mathrm{a}, \mathrm{b}]$ de $[0,1]$. Não é óbvio como argumentos de utilidade epistêmica podem ser estendidos para valores imprecisos de graus de crença. Para mais informações, ver Conor Mayo-Wilson \& Gregory Wheeler (2016).

7 Versões alternativas de axiomatização do cálculo de probabilidades estão disponíveis em Jonathan Weisberg (2011).

${ }^{8}$ Conjunto finito (aditividade finita) ou infinito contável (aditividade contável).

${ }^{9}$ Quando duas ou mais proposições são mutuamente exclusivas ou incompatíveis, apenas uma delas pode ser verdadeira. Portanto, a probabilidade da sua conjunção é zero.
}

\begin{tabular}{|c|c|c|c|c|c|}
\hline intuitio & $\begin{array}{c}\text { ISSN } \\
1983-4012\end{array}$ & Porto Alegre & Vol.10 $-\mathrm{N}^{\circ} .1$ & $\begin{array}{l}\text { Julho } \\
2017\end{array}$ & p.15-24 \\
\hline
\end{tabular}


Teoria de Utilidade Epistêmica e o Argumento da Acurácia a favor do Probabilismo

Assumindo o cálculo proposicional como a lógica de $\mathcal{F}$, é possível derivar um amplo conjunto de teoremas de tais axiomas. ${ }^{10}$ Portanto, as tautologias e contradições lógicas assumidas em (2a) e (2b) são as tautologias e contradições usuais do cálculo proposicional, o que torna nosso conjunto de axiomas suficientemente fraco. Não precisamos entrar em detalhes sobre as consequências lógicas dos axiomas acima. É importante observar que, além de 0 ser o limite inferior, precisamos assumir que 1 é o limite superior do intervalo valorado em números reais.

\section{Teoria de Utilidade Epistêmica}

É correto dizer que o argumento original de Joyce (1998) inaugurou um novo tipo de estratégia de justificação de princípios e normas epistêmicas. A sua estratégia faz parte de um contexto mais amplo e permitiu o desenvolvimento de uma área de pesquisa consolidada recentemente: conhecida como teoria de utilidade epistêmica ou teoria da decisão epistêmica, que é liderada atualmente por Richard Pettigrew. ${ }^{11}$

Argumentos de utilidade ou desutilidade epistêmica usam o aparato formal que é característico das teorias normativas da decisão ou escolha racional. Estas últimas se ocupam estritamente de normas que impõem condições de racionalidade pragmática para ações. Considerando um conjunto $\Omega$ de mundos possíveis e uma partição $\mathrm{O}$ de ações disponíveis a um agente, uma função de utilidade toma como inputs as consequências de uma determinada ação $A \in \mathrm{O}$, que o agente pode decidir realizar, e cada mundo $\omega_{i} \in$ $\Omega$ e retorna como output um resultado que mede o valor de se realizar $A$ em cada $\omega_{i}$. Assim, por exemplo, para quaisquer ações $A, B \in \mathrm{O}$, um princípio de racionalidade pragmática diz que um agente racional $S$ não deve realizar $B$ se $A$ tem maior valor de utilidade do que $B$ para qualquer mundo $\omega_{i} \in \Omega$. Esse princípio é conhecido como dominância forte ${ }^{12}$ e veremos a sua versão epistêmica na próxima seção.

No entanto, em vez de tomar uma ação disponível que o agente pode realizar, medidas de utilidade epistêmica tomam como input um estado epistêmico que o agente pode estar, preservando o conjunto de mundos possíveis na função de utilidade, e retornam um valor como output. Ou seja, sob uma interpretação epistêmica, a função de utilidade (ou desutilidade) toma estados epistêmicos e os mundos de

\footnotetext{
${ }^{10}$ Por exemplo, assumindo que $\operatorname{cr}(\bullet)$ é uma função de probabilidade definida sobre $\mathcal{F}, \operatorname{cr}(p) \leq 1, \operatorname{cr}(p) \geq \operatorname{cr}(p \wedge r)$, $\operatorname{cr}(p) \leq \operatorname{cr}(q)$ quando $p$ acarreta $q$ e $\operatorname{cr}(p)=\operatorname{cr}(r)$ quando $p \equiv r$.

11 Richard Pettigrew coordena um projeto em teoria de utilidade epistêmica financiado pelo European Research Council. Em linhas gerais, a sua pesquisa consiste em aplicar métodos, ferramentas formais e princípios de teoria da decisão para derivar teoremas e argumentos a favor de normas epistêmicas. Mais informações sobre a sua pesquisa e os resultados já alcançados estão disponíveis em http://sites.google.com/site/epistemicutilitytheorybristol/home. No seu livro recente Accuracy and the Laws of Credence (2016), Pettigrew desenvolve uma proposta detalhada de teoria de utilidade epistêmica cuja principal medida de utilidade é a acurácia. Além disso, ele investiga as principais tentativas de justificação de normas e princípios Bayesianos que orientam e governam graus de crença. O seu livro certamente estabelece uma agenda para pesquisas futuras na área de teoria de utilidade epistêmica e epistemologia formal.

12 Ver Richard Pettigrew (2016: 9).
}

\begin{tabular}{|c|c|l|l|l|l|}
\hline intuitio & $\begin{array}{c}\text { ISSN } \\
1983-4012\end{array}$ & Porto Alegre & Vol.10 $-\mathrm{N}^{\circ} .1$ & $\begin{array}{l}\text { Julho } \\
2017\end{array}$ & p.15-24 \\
\hline
\end{tabular}


$\Omega$ como inputs e retorna como output um valor estritamente epistêmico. Substitui-se, portanto, o conjunto de ações disponíveis a $S$ por um conjunto de estados epistêmicos possíveis a $S .{ }^{13}$ Tipicamente, qualquer valor real $\chi \in[0,1]$ de crença gradual constitui o item de entrada em relação ao estado epistêmico, ao passo que o valor (ou desvalor) epistêmico produzido geralmente é de acurácia (inacurácia) ou calibragem.

De acordo com Richard Pettigrew (2013: 899 e 2015: sec. 2), três componentes constituem a estrutura geral de estratégias baseadas em teoria de utilidade epistêmica: ( $i$ ) uma função de utilidade interpretada epistemicamente, (ii) um princípio ou norma de teoria da decisão e (iii) um teorema matemático que possibilita aplicar (ii) em (i) e deduzir, em conjunto com outras suposições, uma norma epistêmica. No nosso caso, a norma PROB da seção anterior. Na verdade, a estratégia que exploraremos neste artigo mostra que a verdade de PROB é alcançada de modo indireto. O teorema matemático da acurácia gradacional nos permite determinar que as funções de graus de crença que violam PROB são irracionais porque têm maior grau de inacurácia do que qualquer função alternativa que satisfaz PROB. ${ }^{14}$ Além do teorema da acurácia gradacional, cuja validade não será desafiada neste artigo, assumiremos a noção de inacurácia como medida de desutilidade e a versão epistêmica do princípio de dominância forte tal qual a defesa de Joyce (1998). O objetivo propriamente epistêmico a ser perseguido é: um agente $S$ deve ter funções de graus de crença com menor grau de inacurácia, ou maior grau de acurácia, do que qualquer outra função alternativa que ele poderia ter. Ademais, Joyce exige um conjunto bastante amplo de axiomas e restrições formais para medidas de inacurácia. Não trataremos de todos os seus aspectos, mas as principais restrições irão aparecer ao longo da próxima seção.

\section{Argumento da Acurácia Gradacional}

Como dissemos, a principal motivação de Joyce (1998) é oferecer um argumento epistêmico em defesa de PROB. Nesse sentido, acurácia-inacurácia gradacional deve desempenhar papel semelhante a outras normas epistêmicas que comumente são fornecidas para o modelo de crença simpliciter. A norma da verdade (NT), por exemplo, impõe um critério de sucesso epistêmico para crenças tudo-ou-nada: o conjunto de crenças de $S$ deve ser tal que no cômputo geral $S$ crê mais em verdades do que em

\footnotetext{
${ }^{13}$ Aqui é importante observar que $S$ pode encontrar-se em algum destes estados epistêmicos, mas não é o caso que $S$ pode escolher estar em algum deles, o que implicaria o voluntarismo doxástico. Agradecimento a um avaliador anônimo por chamar atenção a esse ponto.

${ }^{14} \mathrm{O}$ teorema da acurácia gradacional, cuja formulação veremos na seção 4, é chamado de teorema principal (main theorem) por Joyce (1998: 597-600).
}

\begin{tabular}{|c|c|c|c|c|c|}
\hline intuitio & $\begin{array}{c}\text { ISSN } \\
1983-4012\end{array}$ & Porto Alegre & Vol.10 $-\mathrm{N}^{\circ} .1$ & $\begin{array}{l}\text { Julho } \\
2017\end{array}$ & p.15-24 \\
\hline
\end{tabular}


falsidades. ${ }^{15}$ De modo análogo, Joyce (1998: 579) formula uma norma geral de racionalidade para graus de crença:

〈NGA〉: O conjunto de graus de crença $\mathcal{A}$ de um agente epistemicamente racional $S$ deve ser tal que o seu grau de acurácia é pelo menos tão alto (a saber, é maior ou é igual) do que o grau de acurácia de qualquer conjunto alternativo de graus de crença $\mathcal{A l}^{\prime}$ que $S$ poderia adotar.

Neste momento, alguns esclarecimentos são bem-vindos. Primeiro, como bem observa Pettigrew (2015: sec. 4 e 2016: 36), existem duas maneiras distintas pelas quais se pode medir acurácia (ou inacurácia) ou, em sentido mais amplo, existem dois modos de se medir utilidade (ou desutilidade) epistêmica. Quando avaliamos a acurácia de uma crença gradual sobre uma determinada proposição em um dado mundo $\omega$, trata-se de uma medida de acurácia local: por exemplo, a acurácia de um grau de crença individual sobre $p$ em $\omega$. Diferentemente, quando avaliamos a acurácia de uma função de graus de crença $\operatorname{cr}(\bullet)$ sobre um conjunto de proposições em $\omega$, trata-se de uma medida de acurácia global. Em outros termos, mede-se a acurácia de $\operatorname{cr}\left(p_{i}\right)$ para todos $p_{i} \in \mathcal{F}$ em $\omega$. Para o propósito atual, vamos nos concentrar em medidas globais, que é o objetivo precípuo de Joyce (1998). ${ }^{16}$ Segundo, além das diferenças entre os dois modelos de crença, há uma diferença crucial entre as normas epistêmicas NT e NGA. O modelo de crença simpliciter supõe dois ou no máximo três valores para atitudes doxásticas: crença, descrença e suspensão de juízo. As maneiras de se estar errado são menores. Quando examinamos graus de crença, como o próprio Joyce (1998: 579) enfatiza, há várias maneiras de se estar epistemicamente errado. Qualquer valor $\chi \neq 1$ é uma maneira de se estar errado sobre uma proposição verdadeira e o mesmo vale para qualquer valor $\chi \neq 0$ para proposições falsas. ${ }^{17}$ Assim, não é desejável epistemicamente ter somente graus de crença com valores extremos, máximo ou mínimo. Em termos globais, valores intermediários são mais desejáveis, pois diminuem o risco de o agente ter um nível de inacurácia muito alto ou um nível de acurácia muito baixo. Evidentemente que inacurácia em grau mínimo e acurácia em grau máximo são as metas ideais. Terceiro, inacurácia e acurácia são medidas definidas em termos graduais, como NGA e outras passagens do nosso texto sugerem. Veremos a seguir como é possível medir formalmente tal propriedade de crenças graduais.

Antes, no entanto, precisamos estabelecer uma suposição basilar da proposta de Joyce (1998):

\footnotetext{
${ }^{15}$ Crer simpliciter em uma proposição verdadeira $p$ é sempre melhor do que não crer que $p$, ao passo que não crer em uma proposição falsa $r$ é sempre melhor do que crer simpliciter que $r$.

${ }^{16}$ Mais sobre medidas locais de utilidade epistêmica em Leitgeb \& Pettigrew (2010).

${ }^{17}$ Ou seja, para valores tais que $\chi \in[0,1)$ e $\chi \in(0,1]$ respectivamente.
}

\begin{tabular}{|c|c|c|c|c|c|}
\hline intuitio & $\begin{array}{c}\text { ISSN } \\
1983-4012\end{array}$ & Porto Alegre & Vol.10 $-\mathrm{N}^{\circ} .1$ & $\begin{array}{l}\text { Julho } \\
2017\end{array}$ & p.15-24 \\
\hline
\end{tabular}


$\langle\mathbf{A C C}\rangle$ : Acurácia (ou inacurácia) gradacional é o modo correto de avaliar a racionalidade epistêmica de graus de crença. ${ }^{18}$

Em sentido rigoroso, acurácia gradacional mede a distância entre funções de graus de crença e funções de atribuições consistentes de valor de verdade. Graus de crença com menor grau de inacurácia (ou maior grau de acurácia) estão mais próximos de tais valores de verdade, graus de crença com maior grau de inacurácia (ou menor grau de acurácia) estão mais longe. Tornando nossa análise ainda mais refinada, assumiremos que $C_{\mathcal{F}}$ é o conjunto de funções de graus de crença sobre uma álgebra $\mathcal{F}$ que um agente $S$ pode adotar. Além disso, vamos considerar que $\mathrm{W}_{\mathcal{F}}$ é o conjunto de mundos possíveis relativos a $\mathcal{F}$, isto é, mundos nos quais as proposições de $\mathcal{F}$ têm um valor de verdade determinado, verdadeiro ou falso. Para quaisquer $\omega \in \mathrm{W}_{\mathcal{F}}$ e $p \in \mathcal{F}$, temos uma função de valor de verdade $V_{\omega}(\bullet)$ tal que:

$$
V_{\omega}(\bullet): \mathcal{F} \rightarrow\{0,1\}
$$

Desse modo, $V_{\omega}(\bullet)$ é uma função que admite somente dois valores: é uma função binária. Se $p$ é verdadeira em $\omega$, então $V_{\omega}(p)=1$. Se $q$ é falsa em $\omega$, então $V_{\omega}(q)=0$. Geralmente, entende-se que $V_{\omega}(\bullet)$ expressa graus de crença ideais ou oniscientes, visto que qualquer função $\operatorname{cr}(\bullet)$ que tem o mesmo valor de $V_{\omega}(\bullet)$ é maximamente acurada ou minimamente inacurada.

Até o momento falamos indiscriminadamente de acurácia e inacurácia. Agora parece apropriado nos concentrarmos mais especificamente na função de desutilidade epistêmica usada por Joyce (1998): inacurácia. Assim, uma função $\mathfrak{J}(c r, \omega)$ vai desempenhar o papel de medir a inacurácia global dos graus de crença de um agente qualquer $S$ em um mundo $\omega$ de $\mathrm{W}_{\mathcal{F}}$. Quanto maior a pontuação de $\mathfrak{I}$, maior será o grau de inacurácia de um conjunto de crenças graduais; quanto menor a pontuação, menor será o seu grau de inacurácia. Maior pontuação significa que maior é a penalidade de um conjunto de graus de crença de $S$ com respeito às unidades de inacurácia gradacional aferidas por $\mathfrak{J}$. Em outras palavras, quanto maior for essa pontuação das crenças graduais de um agente, maior será a distância de tais crenças em relação à verdade das proposições acreditadas. Em termos de inacurácia global, o ideal é uma penalidade nula ou um score com valor 0 na função $\mathfrak{I}$.

Duas restrições ou propriedades são significativamente importantes para a noção de inacurácia de Joyce. A primeira delas diz que $\mathfrak{J}(c r, \omega)$ é uma função contínua não-negativa e o seu valor aumenta à proporção que as crenças de $S$ se tornam mais distantes dos valores determinados por $V_{\omega}(\bullet)$. Dessa maneira, $\mathfrak{I}$ é uma função que toma como itens de entrada $\operatorname{cr}(\bullet) \in \mathrm{C}_{\mathcal{F}} \mathrm{e} \omega \in \mathrm{W}_{\mathcal{F}}$ e, por sua vez, retorna

${ }^{18}$ Pettigrew (2015: sec. 5.1 e 2016: 6-8) chama essa tese de credal veritism, seguindo, sob certo aspecto, o programa veritista em epistemologia de Alvin Goldman (2002: 52-59).

\begin{tabular}{|c|c|l|l|l|l|}
\hline intuitio & $\begin{array}{c}\text { ISSN } \\
1983-4012\end{array}$ & Porto Alegre & Vol.10 $-\mathrm{N}^{\circ} .1$ & $\begin{array}{l}\text { Julho } \\
2017\end{array}$ & p.15-24 \\
\hline
\end{tabular}


como item de saída um valor $\chi \in[0, \infty] .{ }^{19}$ Essa propriedade é chamada de estrutura por Joyce (1998: 591). A segunda delas estabelece que $\mathfrak{I}(c r, \omega)$ é uma função que mede unicamente a diferença entre atribuições consistentes de valor de verdade a proposições de $\mathcal{F}$ para mundos de $\mathrm{W}_{\mathcal{F}}$ e os graus de crença que $\operatorname{cr}(\bullet)$ confere a proposições de $\mathcal{F}$. Por assim dizer, o que importa é o quão bem os graus de crença de um agente representam certos estados ou fatos do mundo. Joyce (1998: 591) denomina essa propriedade de extensionalidade.

A princípio, várias medidas de inacurácia poderiam realizar o papel de $\mathfrak{I}(c r, \omega)$, contanto que cumpram com as condições formais impostas por Joyce (1998: 591-596). Como dissemos, não vamos entrar em detalhes sobre todas tais restrições, tampouco propor uma nova medida de inacurácia ou desutilidade epistêmica. Uma medida em especial, contudo, é uma forte candidata a desempenhar tal tarefa. Ela é conhecida como Brier score e foi proposta originalmente pelo meteorologista Glenn W. Brier (1950) em um contexto distinto do nosso: a análise da acurácia-inacurácia de previsões do tempo. Presumivelmente, ela é definida com a seguinte formulação:

$$
\mathcal{B}(c r, \omega)=\sum_{p_{i} \in \mathcal{F}}\left|v_{\omega}\left(p_{i}\right)-\operatorname{cr}\left(p_{i}\right)\right|^{2}
$$

O Brier score é o primeiro aspecto da estratégia de utilidade ou desutilidade epistêmica que falamos na seção anterior, uma vez que pretende medir inacurácia. ${ }^{20}$ Por seu turno, o segundo aspecto de tal estratégia diz respeito ao princípio ou norma de teoria da decisão. Sob uma interpretação epistêmica, o princípio de teoria da decisão empregado por Joyce (1998) é conhecido como princípio de dominância forte:

$\langle\mathbf{D M F}\rangle$ : Um agente racional $S$ não deve ter uma função $\operatorname{cr}(\bullet)$ se há uma função alternativa $\operatorname{cr}{ }^{\prime}(\bullet)$ com pontuação (score) menor para todos os mundos $\omega \in \mathrm{W}_{\mathcal{F} .}{ }^{21}$

\footnotetext{
${ }^{19}$ Este intervalo contém qualquer valor real não-negativo e $\infty$, isto é, forma o conjunto $\mathbb{R} U\{+\infty\}$. Esta condição permite que um conjunto de crenças graduais seja infinitamente inacurado. Embora à primeira vista pareça contraintuitiva, uma razão para adotá-la, como o fazem Joyce (1998: 591) e Pettigrew (2016: 37 e 51), diz respeito à natureza matemática das principais medidas de inacurácia. Algumas delas, como a medida logarítmica aditiva, violam a condição alternativa onde $\mathfrak{J}(c r, \omega)<\infty$, a saber, para qualquer valor real $\chi \in[0, \infty)$. A despeito de darmos prioridade ao Brier score, que é considerada a medida padrão, deixamos em aberto a questão sobre quais das medidas disponíveis no mercado são mais apropriadas para medir inacurácia ou acurácia gradacional. Agradecimento a um parecerista anônimo por exigir mais explicações a respeito de tal restrição.

${ }^{20}$ Trata-se da distância Euclidiana ao quadrado entre $\operatorname{V} \omega(\bullet)$ e $\operatorname{cr}(\bullet)$, a saber, $\mathcal{B}(c r, \omega):=\mathfrak{D}^{2}(V \omega, c r)$. Vale ressaltar que enquanto $\mathcal{B}(c r, \omega)$ mede inacurácia, o seu negativo, $-\mathcal{B}(c r, \omega)$, mede acurácia.

${ }^{21}$ Ver Pettigrew (2015: sec. 5.1 e 2016: 9). Também chamado de princípio de dominância simples ou ingênua.
}

\begin{tabular}{|c|c|l|l|l|l|}
\hline intuitio & $\begin{array}{c}\text { ISSN } \\
1983-4012\end{array}$ & Porto Alegre & Vol.10 $-\mathrm{N}^{\circ} .1$ & $\begin{array}{l}\text { Julho } \\
2017\end{array}$ & p.15-24 \\
\hline
\end{tabular}


O princípio DMF enuncia que para quaisquer funções $\operatorname{cr}(\bullet)$ e $\operatorname{cr}^{\prime}(\bullet)$ e um agente $S$, se $S$ adota $c r(\bullet)$ e $\mathfrak{I}(c r, \omega)>\mathfrak{I}\left(c r^{\prime}, \omega\right)$ é o caso para todos os mundos $\omega$ de $\mathrm{W}_{\mathcal{F}}$, então $S$ é irracional. Portanto, $c r^{\prime}(\bullet)$ domina fortemente $\operatorname{cr}(\bullet)$ porque a primeira função tem menor grau de inacurácia, tem desutilidade epistêmica menor, do que a segunda para todos os mundos. Se empregarmos o Brier score, significa que a pontuação de $\mathcal{B}(c r, \omega)$ é maior do que a pontuação de $\mathcal{B}\left(c r^{\prime}, \omega\right)$ para qualquer mundo de $\mathrm{W}_{\mathcal{F}}$ :

$$
\sum_{p_{i} \in \mathcal{F}}\left|v_{\omega}\left(p_{i}\right)-c r\left(p_{i}\right)\right|^{2}>\sum_{p_{i} \in \mathcal{F}}\left|v_{\omega}\left(p_{i}\right)-c r^{\prime}\left(p_{i}\right)\right|^{2}
$$

O terceiro fator da proposta de Joyce (1998) é um teorema matemático. Podemos chamá-lo de teorema da acurácia gradacional. Como dissemos, $\mathfrak{I}$ é um score que mede inacurácia e cujos valores podem ser qualquer membro de $\mathbb{R} U\{+\infty\}$. Além disso, $\mathfrak{I}$ é uma função cujo domínio é o produto cartesiano $\mathrm{C}_{\mathcal{F}} \times \mathrm{W}_{\mathcal{F} .}{ }^{22}$ Assim, o teorema da acurácia gradacional ou o teorema principal de Joyce (1998: 597-600) pode ser formulado nos seguintes termos:

$\langle$ GAT $\rangle:$ Assumindo que $\mathfrak{J}: \mathrm{C}_{\mathcal{F}} \times \mathrm{W}_{\mathcal{F}} \rightarrow[0, \infty]$ é uma medida de inacurácia apropriada, se $\operatorname{cr}(\bullet)$ de um agente $\mathrm{S}$ viola o cálculo de probabilidades, então há uma função alternativa $c r^{\prime}(\bullet)$ que satisfaz o cálculo de probabilidades, onde ambas $\operatorname{cr}(\bullet)$ e $c r^{\prime}(\bullet) \in \mathrm{C}_{\mathcal{F}}$, tal que $\mathfrak{I}\left(c r^{\prime}, \omega\right)<\mathfrak{I}(c r, \omega)$ para qualquer $\omega \in \mathrm{W}_{\mathcal{F} .}{ }^{23}$

Se $\mathfrak{J}(c r, \omega)>\mathfrak{J}\left(c r^{\prime}, \omega\right)$ é o caso, dizemos que $c r^{\prime}(\bullet)$ domina $c r(\bullet)$, uma vez que $c r^{\prime}(\bullet)$ tem menor pontuação do que $\operatorname{cr}(\bullet)$ para todos os mundos. GAT determina que funções não probabilísticas de crença são dominadas fortemente por funções probabilísticas. Por limitação de espaço, não vamos reconstruir a demonstração para GAT, tampouco desafiá-lo. ${ }^{24}$ Todavia, podemos fornecer um cenário no qual aplicamos o Brier score sobre diferentes conjuntos de graus de crença, mostrando que um conjunto que satisfaz PROB domina fortemente conjuntos que não o satisfazem.

Vamos assumir uma álgebra $\mathcal{F}=\{p, \neg p\}$ e um conjunto de dois mundos possíveis relativos a $\mathcal{F}$ tal que $\mathrm{W}_{\mathcal{F}}=\left\{\omega_{1}, \omega_{2}\right\}$. Suponhamos, adicionalmente, que $p$ é verdadeira em $\omega_{1}, V_{\omega 1}(p)=1$ e $V_{\omega 1}(\neg p)=$ 0 , e $\neg p$ é verdadeira em $\omega_{2}, V_{\omega 2}(p)=0$ e $V_{\omega 2}(\neg p)=1$. Para ilustrarmos melhor, considere a figura 1 abaixo. A reta A-B em cor vermelha representa as funções de graus de crença que obedecem aos axiomas de probabilidade. Qualquer ponto fora dessa reta representa alguma função não-probabilística de graus de

${ }^{22}$ Ou seja, $C_{\mathcal{F}} \times \mathrm{W}_{\mathcal{F}}=\left\{(\mathrm{a}, \mathrm{b}) \mid \mathrm{a} \in \mathrm{C}_{\mathcal{F}} \wedge \mathrm{b} \in \mathrm{W}_{\mathcal{F}}\right\}$.

${ }^{23}$ Ver Joyce (1998: 597-598), Hájek (2009: 243) e Pettigrew (2015: sec. 5.1).

${ }^{24}$ Para uma contestação das restrições e axiomas requeridos para GAT, ver Patrick Maher (2002).

\begin{tabular}{|c|c|l|l|l|l|}
\hline intuitio & $\begin{array}{c}\text { ISSN } \\
1983-4012\end{array}$ & Porto Alegre & Vol.10 $-\mathrm{N}^{\circ} .1$ & $\begin{array}{l}\text { Julho } \\
2017\end{array}$ & p.15-24 \\
\hline
\end{tabular}


crença. O ponto A representa o grau de crença ideal de que $p$ em $\omega_{1}$ e o ponto B o grau de crença ideal de que $\neg p$ em $\omega_{2}$.

\section{Figura 1:}

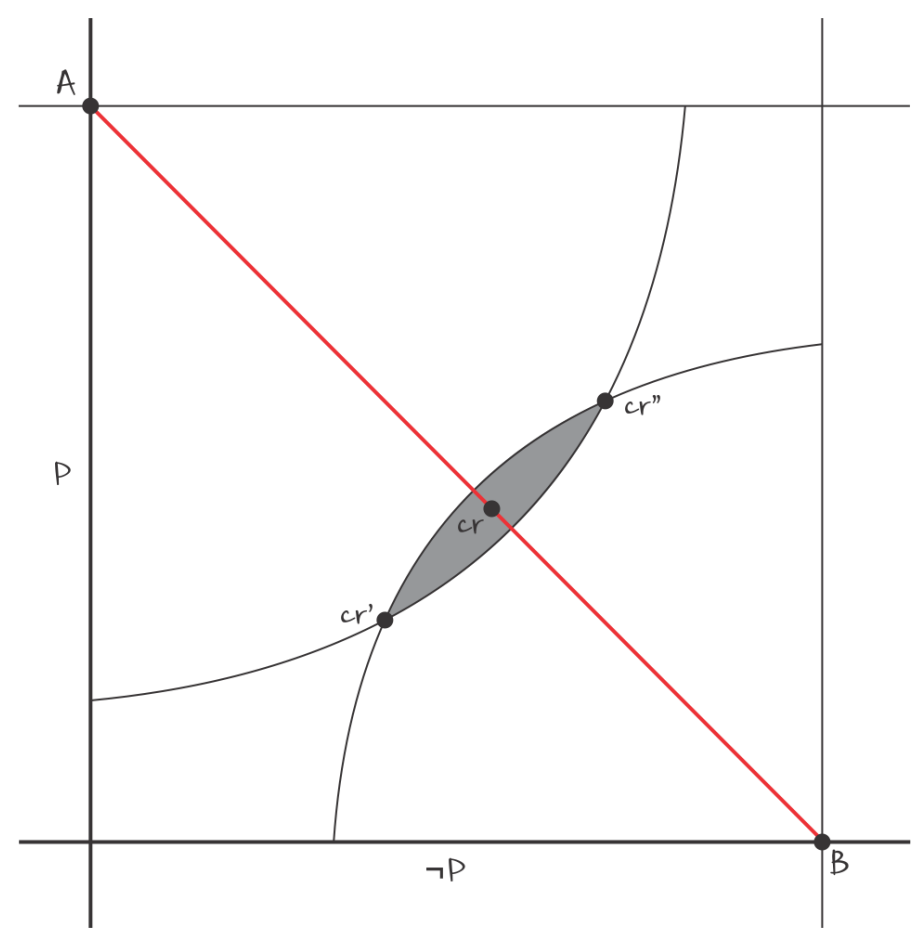

$\mathrm{Na}$ figura acima, temos três funções de crença sobre $p$ e $\neg p: \operatorname{cr}(\bullet), \operatorname{cr}{ }^{\prime}(\bullet)$ e $c r r^{\prime \prime}(\bullet)$. Vamos considerar os seguintes valores abaixo para essas três funções, a saber, funções de graus de crença distintas de $\mathrm{C}_{\mathcal{F}}$ sobre as proposições $p, \neg p \in \mathcal{F}$. Mostraremos que, aplicando o Brier score, $\operatorname{cr}(\bullet)$ domina $c r^{\prime}(\bullet)$ e $c r^{\prime ’}(\bullet)$ para ambos mundos $\omega_{1}, \omega_{2} \in \mathrm{W}_{\mathcal{F}}$.
(A) $1=\left\{\begin{array}{c}c r(p)=0.45 \\ + \\ \operatorname{cr}(\neg p)=0.55\end{array}\right.$
(B) $1>\left\{\begin{array}{c}c r^{\prime}(p)=0.3 \\ + \\ c r^{\prime}(\neg p)=0.4\end{array}\right.$

\begin{tabular}{|c|c|c|c|c|c|}
\hline intuitio & $\begin{array}{c}\text { ISSN } \\
1983-4012\end{array}$ & Porto Alegre & Vol.10- No.1 & $\begin{array}{l}\text { Julho } \\
2017\end{array}$ & p.15-24 \\
\hline
\end{tabular}




$$
\text { (C) } 1<\left\{\begin{array}{c}
c r^{\prime \prime}(p)=0.6 \\
+ \\
c r^{\prime \prime}(\neg p)=0.7
\end{array}\right.
$$

As funções em (A) estão de acordo com PROB, ao passo que $(\mathbf{B})$ e $(\mathbf{C})$ não estão. O conjunto em (A) obedece aos axiomas de aditividade e de normalização que estabelecemos na seção 2. Portanto, (A) é uma função probabilística: $p$ e $\neg p$ são incompatíveis, a probabilidade de ( $p \vee \neg p$ ) deve ser igual à soma dos seus disjunctos e a probabilidade de uma tautologia deve ser igual a 1. Observe que os conjuntos (B) e (C) violam o axioma de normalização. A probabilidade $(p \vee \neg p)$ é menor do que $1 \mathrm{em}(\mathbf{B})$ e maior do que 1 em $(\mathbf{C})$. Como dissemos, $\operatorname{cr}(\bullet)$ domina $c r^{\prime}(\bullet)$ e $c r$ '’ $(\bullet)$ para todos os mundos de $\mathrm{W}_{\mathcal{F}}$. Se empregarmos o Brier score, veremos que os graus de inacurácia da função $\operatorname{cr}(\bullet)$ são $\mathcal{B}\left(c r, \omega_{1}\right) \approx 0.6 \mathrm{e} \mathcal{B}\left(c r, \omega_{2}\right) \approx 0.4$ :

$$
\text { (A) }\left\{\begin{array}{l}
\left|v_{\omega 1}(p)-\operatorname{cr}(p)\right|^{2}+\left|v_{\omega 1}(\neg p)-\operatorname{cr}(\neg p)\right|^{2}=|1-0.45|^{2}+|0-0.55|^{2} \approx 0.6 . \\
\left|v_{\omega 2}(p)-\operatorname{cr}(p)\right|^{2}+\left|v_{\omega 2}(\neg p)-\operatorname{cr}(\neg p)\right|^{2}=|0-0.45|^{2}+|1-0.55|^{2} \approx 0.4 .
\end{array}\right.
$$

Ambas $c r^{\prime}(\bullet)$ e $c r ’(\bullet)$ têm maior grau de inacurácia do que $\operatorname{cr}(\bullet)$ em aproximadamente 0.05 para qualquer mundo $\omega_{i} \in \mathrm{W}_{\mathcal{F}}$. Para ver isso, basta comparar os valores de inacurácia de (A) com os valores de (B) e (C) para os mundos de $\mathrm{W}_{\mathcal{F}}$ :
(B) $\left\{\begin{array}{l}\left|v_{\omega 1}(p)-c r^{\prime}(p)\right|^{2}+\left|v_{\omega 1}(\neg p)-c r^{\prime}(\neg p)\right|^{2}=|1-0.3|^{2}+|0-0.4|^{2}=0.65 . \\ \left|v_{\omega 2}(p)-c r^{\prime}(p)\right|^{2}+\left|v_{\omega 2}(\neg p)-c r^{\prime}(\neg p)\right|^{2}=|0-0.3|^{2}+|1-0.4|^{2}=0.45 .\end{array}\right.$
(C) $\left\{\begin{array}{l}\left|v_{\omega 1}(p)-c r^{\prime \prime}(p)\right|^{2}+\left|v_{\omega 1}(\neg p)-c r^{\prime \prime}(\neg p)\right|^{2}=|1-0.6|^{2}+|0-0.7|^{2}=0.65 . \\ \left|v_{\omega 2}(p)-c r^{\prime \prime}(p)\right|^{2}+\left|v_{\omega 2}(\neg p)-c r^{\prime \prime}(\neg p)\right|^{2}=|0-0.6|^{2}+|1-0.7|^{2}=0.45 .\end{array}\right.$

Os graus de inacurácia de $(\mathbf{B})$ e $(\mathbf{C})$ são: $\mathcal{B}\left(c r^{\prime}, \omega_{1}\right)=0.65$ e $\mathcal{B}\left(c r^{\prime}, \omega_{2}\right)=0.45 ; \mathcal{B}\left(c r^{\prime \prime}, \omega_{1}\right)=0.65$ e $\mathcal{B}\left(c r\right.$ '”, $\left.\omega_{2}\right)=0.45$. Portanto, o conjunto (A) tem menor grau de inacurácia do que os conjuntos $(\mathbf{B})$ e (C); as duas últimas têm o mesmo grau de inacurácia em ambos os mundos. Ademais, qualquer ponto na área em cinza entre os dois arcos da figura 1 acima, área que inclui $\operatorname{cr}(\bullet)$, tem uma pontuação menor portanto, tem menor grau de inacurácia -, do que $c r^{\prime}(\bullet)$ e $c r^{\prime \prime}(\bullet)$ em ambos os mundos $\omega_{1}$ e $\omega_{2}$. As funções $c r^{\prime}(\bullet)$ e $c r '(\bullet)$ são dominadas fortemente por qualquer ponto de tal área.

Neste momento, podemos reconstruir o argumento da acurácia gradacional de Joyce (1998) com a seguinte formulação:

\begin{tabular}{|c|c|l|l|l|l|}
\hline intuitio & $\begin{array}{c}\text { ISSN } \\
1983-4012\end{array}$ & Porto Alegre & Vol.10 $-\mathrm{N}^{\circ} .1$ & $\begin{array}{l}\text { Julho } \\
2017\end{array}$ & p.15-24 \\
\hline
\end{tabular}


(P1) ACC. Acurácia (ou inacurácia) gradacional é o modo correto de avaliar a racionalidade epistêmica de graus de crença.

(P2) GAT. Se $\operatorname{cr}(\bullet)$ de um agente $S$ viola o cálculo de probabilidades, então há uma função alternativa $c r^{\prime}(\bullet)$ que o satisfaz e domina (fortemente) $\operatorname{cr}(\bullet), \mathcal{B}\left(c r^{\prime}, \omega_{i}\right)<\mathcal{B}\left(c r, \omega_{i}\right)$, para qualquer mundo $\omega_{i} \in \mathrm{W}_{\mathcal{F}}$.

(P3) DMF. Se $\operatorname{cr}(\bullet)$ é dominada fortemente por uma função alternativa $c r(\bullet)$ menos inacurada, então $S$ é irracional.

(C) $\therefore$ Se a função $\operatorname{cr}(\bullet)$ de um agente $S$ viola o cálculo de probabilidades, então $S$ é irracional. ${ }^{25}$

O argumento acima é dedutivamente válido. Como falamos anteriormente, PROB se segue do argumento de Joyce (1998) de modo indireto. Agentes que violam o cálculo de probabilidades são considerados irracionais. ${ }^{26}$ Embora o argumento seja bastante atraente, recentemente alguns problemas contundentes foram oferecidos contra ele na literatura sobre epistemologia formal. Vamos explorar três deles na próxima seção.

\section{Três Problemas para o Argumento da Acurácia Gradacional}

Nesta seção, discutiremos três problemas que foram apresentados contra o argumento original de Joyce (1998). De todo modo, Joyce (2009) forneceu uma versão mais sofisticada do seu argumento, respondendo a vários problemas e objeções. Devido à sua complexidade e aos seus detalhes técnicos, não tratamos dessa nova versão, deixando-a para uma próxima oportunidade.

Um problema interessante foi sugerido por Alan Hájek (2009: 245). Suponha que $S$ mantém algum conjunto de graus de crença que não estão de acordo com PROB. Por exemplo, $c r^{\prime}(\bullet)$ ou $c r{ }^{\prime \prime}(\bullet)$ da seção anterior. Elas têm maior grau de inacurácia do que uma outra função qualquer $c r^{*}(\bullet)$, que também é não probabilística. Basta observar que existem outros pontos da área em cor cinza da figura 1 que, embora não estejam situados na reta A-B, dominam $c r^{\prime}(\bullet)$ e $c r^{\prime}(\bullet)$. A função $c r^{*}(\bullet)$ desempenha esse papel. Nesse sentido, é um desideratum que um agente $S$ deve se mover de $c r^{\prime}(\bullet)$ ou $c r^{\prime \prime}(\bullet)$ para uma função com menor grau de inacurácia, como é o caso de $c r^{*}(\bullet)$. Mas, afinal, esse não poderia ser o ponto de parada estável para $S$ ? Por que $S$ deve se mover de $c r^{\prime}(\bullet)$ para uma outra função, uma que seja probabilística? Claramente, pode-se responder que, por sucessivas aplicações de GAT, funções nãoprobabilísticas seriam sempre dominadas em termos de Brier score por funções probabilísticas. No

\footnotetext{
${ }^{25}$ Reconstrução semelhante em Richard Pettigrew (2015: sec. 5.1) e Darren Bradley (2015, cap. 3).

${ }^{26} \mathrm{O}$ contrapositivo da conclusão diz que se $S$ não é irracional, então a função $\operatorname{cr}(\bullet)$ de $S$ não viola o cálculo de probabilidades.
}

\begin{tabular}{|c|c|c|c|c|c|}
\hline intuitio & $\begin{array}{c}\text { ISSN } \\
1983-4012\end{array}$ & Porto Alegre & Vol.10 $-\mathrm{N}^{\circ} .1$ & $\begin{array}{l}\text { Julho } \\
2017\end{array}$ & p.15-24 \\
\hline
\end{tabular}


entanto, talvez Hájek esteja exigindo uma razão mais forte para que uma função probabilística de graus de crença seja o ponto de parada estável de $S$, mas sem apelo à recursividade de GAT. Um teorema da acurácia gradacional converso (CGAT), como o próprio Hájek (2009: 245) observa, poderia desempenhar essa tarefa mais diretamente: se $\operatorname{cr}(\bullet)$ de $S$ satisfaz o cálculo de probabilidades, então não há uma função não-probabilística $c r^{\prime}(\bullet)$ que domina $\operatorname{cr}(\bullet)$ para qualquer mundo $\omega_{i} \in \mathrm{W}_{\mathcal{F}}$. Joyce (2009) parece indicar que esse teorema é demonstrável, o que pode oferecer uma prova mais direta para justificação de PROB.

Um problema levantado por Easwaran e Fitelson (2012) sugere um conflito entre diferentes tipos de normas epistêmicas para crenças graduais: entre a norma da evidência $(\mathbf{E N})$ e o que nós chamamos de ACC. Em linhas gerais, EN diz que graus de crença de um agente racional $S$ devem corresponder à evidência que $S$ dispõe. Além da meta epistêmica de se estar próximo da verdade, agentes racionais devem proporcionar as suas crenças de acordo com a sua evidência. Tipicamente reprovamos agentes que não respondem à evidência de maneira apropriada, enquanto aprovamos agentes que o fazem. Nesse sentido, crer conforme a evidência que se dispõe parece ser epistemicamente desejável. Observe que NGA não diz nada sobre a acurácia ser o único ou o mais importante valor que deve ser considerado quando avaliamos o status epistêmico de graus de crença. Por sua vez, ACC sugere isso. Uma das críticas de Easwaran e Fitelson consiste em um caso no qual há um conflito entre ACC e uma versão do princípio principal (PP) de Lewis (1980), o qual diz que probabilidades subjetivas (graus de crença) devem corresponder a probabilidades físicas ou objetivas (chance) quando estas são evidência para o agente. Porém, segundo eles, isso pode ser estendido para um problema mais geral onde EN e ACC não estão em sintonia. Podemos ilustrar esse problema com um exemplo. ${ }^{27}$

Suponhamos que $S$ sabe que uma determinada moeda é enviesada para o resultado de coroa quando jogada. Para duas possibilidades de resultado, cara ou coroa, vamos assumir que a probabilidade objetiva de sair coroa é 0.8 corresponde à evidência total de $S$. Embora $S$ proporcione o seu grau de crença de acordo com a sua evidência a favor de obter o resultado de coroa, $S$ não está de acordo com PROB, uma vez que atribui grau de crença 0.7 ao resultado de cara. Portanto, cr(cara $\vee$ coroa $)>1$. Acontece, todavia, que nenhuma outra função probabilística alternativa $c r^{*}(\bullet)$ que domina as funções de $S$ em termos de Brier score corresponde à evidência disponível a $S$ : em tais circunstâncias, $c r^{*}$ (cara $\vee$ coroa $)=1, c r^{*}(\operatorname{coroa}) \neq 0.8$ e $c r^{*}(\bullet)$ domina $\operatorname{cr}(\bullet)$. Isto é, das funções probabilísticas que dominam $\operatorname{cr}(\bullet)$, nenhuma satisfaz PP e EN. Por seu turno, a única função que satisfaz PROB, PP e EN não domina $\operatorname{cr}(\bullet)$. Vamos chamar esta última função de $c r^{* *}(\bullet)$. Assumindo que $V_{\omega 1}($ cara $)=1$ e $V_{\omega 2}($ coroa $)=1$, temos $\mathcal{B}\left(c r, \omega_{1}\right)=0.73$ e $\mathcal{B}\left(c r, \omega_{2}\right)=0.53$. Por exemplo, uma função probabilística que domina $\operatorname{cr}(\bullet)$ em todos os mundos é formada pelas seguintes atribuições: $c r^{*}($ cara $)=0.4$ e $c r^{*}($ coroa $)=0.6$, pois $\mathcal{B}\left(c r^{*}, \omega_{1}\right)=$ 0.72 e $\mathcal{B}\left(c r^{*}, \omega_{2}\right)=0.32$. O único conjunto de crenças graduais que satisfaz PROB, PP e EN é ${ }^{27}$ Ver Pettigrew (2014a: 584-585, 2015: sec. 5.4 e 2016: 26-28).

\begin{tabular}{|c|c|l|l|l|l|}
\hline intuitio & $\begin{array}{c}\text { ISSN } \\
1983-4012\end{array}$ & Porto Alegre & Vol.10 $-\mathrm{N}^{\circ} .1$ & $\begin{array}{l}\text { Julho } \\
2017\end{array}$ & p.15-24 \\
\hline
\end{tabular}


constituído pelas seguintes atribuições: $c r^{* *}(\operatorname{cara})=0.2$ e $c r^{* *}(\operatorname{coroa})=0.8$. Entretanto, $c r^{* *}(\bullet)$ não domina $\operatorname{cr}(\bullet)$ em todos os mundos, uma vez que os seus valores de inacurácia são $\mathcal{B}\left(c r^{* *}, \omega_{1}\right)=1.28$ e $\mathcal{B}\left(c r^{* *}, \omega_{2}\right)=0.08$.

Não obstante $S$ obedeça a EN e satisfaça PP, a questão é se $S$ deve ser considerado irracional porque tem crenças com maior grau de inacurácia (ou menor grau de acurácia) do que $c r^{*}(\bullet)$. De fato, é muito forte afirmar que $S$ é epistemicamente irracional se não satisfaz PROB e ACC, considerando outros tipos de objetivos epistêmicos a serem perseguidos. Talvez uma versão de probabilismo mitigado seja simplesmente dizer que obedecer ao cálculo de probabilidades é parte da racionalidade de agentes com crenças graduais, mas não é toda história, o que enfraquece ACC: a alegação de que a acurácia não é a única meta epistêmica a ser buscada. Outro problema é se tais normas e tipos distintos de metas são compatíveis entre si ou se eventualmente deve-se abandonar parte delas. Pettigrew (2014a, 2014b e 2016), por exemplo, considera atraente um programa de accuracy-only epistemology, onde acurácia é o único valor epistêmico para crenças graduais e outros valores podem ser reduzidos a ela. Isso parece comprometê-lo com a tese do monismo de valor cognitivo ou epistêmico. Assim, o ponto é mostrar como é possível compatibilizar as normas da evidência e da acurácia. Adicionalmente, seria preciso fornecer um argumento para a alegação de que satisfazer o valor de ter crenças graduais que sejam proporcionais às evidências que o agente dispõe pode ser reduzido ao valor da acurácia, o que significa mostrar que perseguir o primeiro sempre equivale a perseguir o último. ${ }^{28}$

Uma última consideração torna o debate sobre os princípios de dominância mais refinado. De acordo com Pettigrew (2015: sec. 5.4 e 2016: 9), podemos fazer uma distinção entre dois conceitos de dominância: forte e fraca. Como vimos, uma função $\operatorname{cr}(\bullet)$ domina fortemente $\operatorname{cr}{ }^{\boldsymbol{\prime}}(\bullet)$ quando $\mathfrak{I}\left(\operatorname{cr}, \omega_{i}\right)<$ $\mathfrak{I}\left(c r^{\prime}, \omega_{i}\right)$ é o caso para cada mundo $\omega_{i} \in \mathrm{W}_{\mathcal{F}}$. Diferentemente, $\operatorname{cr}(\bullet)$ domina fracamente $c r^{\prime}(\bullet)$ quando $\mathfrak{I}\left(c r, \omega_{i}\right) \leq \mathfrak{I}\left(c r^{\prime}, \omega_{i}\right)$ é o caso para cada mundo $\omega_{i}$ e $\mathfrak{I}\left(c r, \omega_{k}\right)<\mathfrak{I}\left(c r^{\prime}, \omega_{k}\right)$ é o caso para algum mundo $\omega_{k}$, considerando que $\omega_{i}, \omega_{k} \in \mathrm{W}_{\mathcal{F}}$. Desse modo, dominância fraca ocorre quando $\operatorname{cr}(\bullet)$ tem desutilidade epistêmica pelo menos tão baixa (menor ou igual) quanto $c r^{\prime}(\bullet)$ em todos os mundos e tem menor desutilidade em algum mundo. Ou seja, dominância fraca permite que funções de grau de crença tenham o mesmo grau de inacurácia em alguns mundos, mas em pelo menos um mundo uma função deve ter maior grau de inacurácia do que uma outra função alternativa. O problema é que o princípio DMF não exclui casos nos quais uma função de graus de crença que domina fortemente uma outra função é dominada fracamente por uma terceira. Nessa esteira, parece bastante plausível que DMF seja substituído por um princípio que evite dominância fraca. O princípio de dominância não-dominada ${ }^{29}$ desempenha esse papel:

\footnotetext{
${ }^{28}$ Agradecimento a um avaliador anônimo pelas sugestões acerca de tal objeção.

${ }^{29}$ Ver Pettigrew (2016: 22).
}

\begin{tabular}{|c|c|c|c|c|c|}
\hline intuitio & $\begin{array}{c}\text { ISSN } \\
1983-4012\end{array}$ & Porto Alegre & Vol.10- No.1 & $\begin{array}{c}\text { Julho } \\
2017\end{array}$ & p.15-24 \\
\hline
\end{tabular}


não é racional para $S$ adotar uma função $c r^{\prime}(\bullet)$ se uma função $\operatorname{cr}(\bullet)$ domina fortemente $\operatorname{cr}(\bullet)$ e $\operatorname{cr}(\bullet)$ não é dominada fracamente por uma função alternativa $c r^{*}(\bullet)$.

Embora o princípio de dominância não-dominada seja atraente, existem outros princípios de dominância interessantes disponíveis no mercado. Pettigrew (2016: 23-26) considera o princípio de dominância imodesta mais apropriado para situações epistêmicas, a saber, quando as opções disponíveis são estados doxásticos. No nosso caso, graus de crença. O princípio de dominância não-dominada ficaria restrito a situações práticas, especialmente quando não há uma função probabilística relevante que nos forneceria uma avaliação das opções em termos de utilidade esperada. Além de ser mais fraca do que este último, dominância imodesta determina que não é racional ter funções não-probabilísticas quando elas são dominadas fortemente por funções probabilísticas, sob a condição de que estas últimas não sejam extremamente modestas - isto é, não têm inacurácia esperada maior do que uma função alternativa de graus de crença - segundo uma determinada medida de inacurácia $\mathfrak{J}$. Inacurácia esperada de uma função de graus crença $\operatorname{cr}(\bullet)$, que não é necessariamente probabilística, relativa a uma outra função (probabilística) $\operatorname{pr}(\bullet)$ é a soma ponderada do seu valor de inacurácia em mundos diferentes tal que a ponderação é fornecida por $\operatorname{pr}(\bullet)$. Acontece que, como Pettigrew (2016: 23) observa, em circunstâncias epistêmicas podemos realizar uma avaliação em termos de inacurácia esperada, uma vez que há pelo menos uma função probabilística entre as dominantes. Dois obstáculos, contudo, dificultam essa manobra. Por um lado, o problema é que PROB se segue de GAT somente se assumirmos DMF, mas não se segue se aceitamos o princípio de dominância imodesta ou de dominância não-dominada. Conforme Pettigrew (2016: 35), seria necessário um teorema alternativo ao teorema GAT de Joyce (1998). Assumindo dominância não-dominada ou dominância imodesta como o princípio de teoria da decisão e o Brier score como a medida de inacurácia, consegue-se PROB via teorema da dominância de Bruno de Finetti (1974): qualquer função não-probabilística de graus de crença é fortemente dominada por uma função probabilística e nenhuma função probabilística de graus de crença é fracamente dominada por qualquer outra função não-probabilística. ${ }^{30} \mathrm{Na}$ verdade, GAT é somente uma versão generalizada da primeira parte do teorema obtido por de Finetti; a segunda parte corresponderia ao que denominamos por CGAT, isto é, o teorema converso de GAT. Por outro lado, ainda que dominância imodesta seja o princípio mais interessante, é preciso oferecer uma motivação e propriamente um argumento a favor da tese de que graus de crença devem ser avaliados em termos da sua inacurácia esperada. Embora dito brevemente, Pettigrew (2016: 26) sugere que um argumento semelhante ao seu argumento a favor de PROB sustentaria tal tese. A despeito da nossa breve discussão nos revelar que DMF precisa ser substituído por um princípio de dominância mais plausível, um exame mais cuidadoso dos seus potenciais candidatos precisa ser

${ }^{30}$ Ver Pettigrew (2016: 17).

\begin{tabular}{|c|c|c|c|c|c|}
\hline intuitio & $\begin{array}{c}\text { ISSN } \\
1983-4012\end{array}$ & Porto Alegre & Vol.10 $-\mathrm{N}^{\circ} .1$ & $\begin{array}{l}\text { Julho } \\
2017\end{array}$ & p.15-24 \\
\hline
\end{tabular}


desenvolvido em uma outra oportunidade, avaliando os prós e contras das alternativas propostas na literatura, o que significa reformular o argumento da acurácia gradacional a favor de PROB.

\section{Comentários finais}

Neste artigo, exploramos uma versão de argumento de utilidade epistêmica: o argumento da acurácia gradacional de Joyce (1998). A sua proposta permitiu o desenvolvimento de uma agenda de pesquisa e novas maneiras de justificação de princípios e normas em epistemologia. Vimos, entretanto, que alguns sérios problemas podem ser oferecidos contra o seu argumento. Mais recentemente, Joyce (2009) forneceu uma versão mais engenhosa do que o seu argumento original, conseguindo lidar com algumas das principais objeções levantadas na literatura. Dos três problemas discutidos, vimos que os dois últimos são os mais contundentes. O princípio DMF precisa ser substituído por alguma versão mais fraca de princípio de dominância, o que implica em fornecer um teorema alternativo a GAT - nomeadamente, o teorema da dominância obtido por de Finetti - e reformular o argumento a favor de PROB fornecido no final da seção 4. Deixamos em aberto, no entanto, a questão sobre qual dos princípios de dominância é a melhor alternativa ao princípio DMF. O problema colocado por Easwaran e Fitelson (2012) nos revela um conflito entre normas epistemicamente relevantes. De qualquer forma, o desafio é encontrar uma maneira de compatibilizá-las em um programa de epistemologia Bayesiana mais amplo. Eventualmente, como Pettigrew (2014a, 2014b e 2016) sugere, talvez haja uma maneira de reduzir diversos valores epistêmicos a somente um, o da acurácia, o que significa aceitar a tese do monismo de valor cognitivo ou epistêmico.

\section{Referências}

BRADLEY, D. A Critical Introduction to Formal Epistemology. London: Bloomsbury, 2015.

BRIER, G. W. Verification of Forecasts Expressed in Terms of Probability. Monthly Weather Review, v. 78, n. 1, p. $1-3,1950$.

CHRISTENSEN, D. Putting Logic in its Place: Formal Constraints on Rational Belief. New York: Oxford University Press, 2004.

EASWARAN, K.; FITELSON, B. An Evidentialist Worry about Joyce's Argument for Probabilism. Dialectica, v. 66, n. 3, p. 425-433, 2012.

FINETTI, B. D. Foresight: Its Logical Laws, its Subjective Sources. In: KYBURG, H.; SMOKLER, H. (Eds.). Studies in Subjective Probability. New York: Wiley, 1964, p. 93-159.

FINETTI, B. D. Theory of Probability, Volume I. New York: Wiley \& Sons, 1974.

FRAASSEN, B. C. V. Calibration: A Frequency Justification for Personal Probability. In: COHEN, R. S.; LAUDAN, L. (Eds.). Physics, Philosophy and Psychoanalysis. Dordrecht: Springer, 1983, p. 295-319.

GOLDMAN, A. Pathways to Knowledge: Private and Public. Oxford: Oxford University Press, 2002.

GREAVES, H.; WALLACE, D. Justifying Conditionalization: Conditionalization Maximizes Expected Epistemic Utility. Mind, v. 115, n. 459, p. 607-632, 2006.

HÁJEK, A. Arguments for - or against - Probabilism? In: HUBER, F.; SCHMIDT-PETRI, C. (Eds.). Synthese Library 342, Degrees of Belief. Dordrecht: Synthese, 2009, p. 229-251.

JOYCE, J. A Nonpragmatic Vindication of Probabilism. Philosophy of Science, v. 65, p. 575-603, 1998.

\begin{tabular}{|c|c|c|c|c|c|}
\hline intuitio & $\begin{array}{c}\text { ISSN } \\
1983-4012\end{array}$ & Porto Alegre & Vol.10 $-\mathrm{N}^{\circ} .1$ & $\begin{array}{l}\text { Julho } \\
2017\end{array}$ & p.15-24 \\
\hline
\end{tabular}


JOYCE, J. Accuracy and Coherence: Prospects for an Alethic Epistemology of Partial Belief. In: HUBER, F.; SCHMIDT-PETRI, C. (Eds.). Synthese Library 342, Degrees of Belief. Dordrecht: Synthese, 2009, p. 263-297.

LANGE, M. Calibration and the Epistemological Role of Bayesian Conditionalization. The Journal of Philosophy, v. 96, n. 6, p. 294-324, 1999.

LEITGEB, H. Reducing Belief Simpliciter to Degrees of Belief. Annals of Pure and Applied Logic, v. 164, n. 12, p. 1338-1389, 2013.

LEITGEB, H.; PETTIGREW, R. An Objective Justification of Bayesianism I: Measuring Inaccuracy. Philosophy of Science, v. 77, n. 2, p. 201-235, 2010.

LEWIS, D. A Subjectivist's Guide to Objective Chance. In: JEFFREY, R. (Ed.). Studies in Inductive Logic and Probability, vol. II. Berkeley, CA: University of California Press, 1980.

MAHER, P. Betting on Theories. Cambridge: Cambridge University Press, 1993.

MAHER, P. Depragmatized Dutch Book Arguments. Philosophy of Science, v. 64, n. 2, p. 291-305, 1997.

MAHER, P. Joyce's Argument for Probabilism. Philosophy of Science, v. 69, n. 1, p. 73-81, 2002.

MAYO-WILSON, C.; WHEELER, G. Scoring Imprecise Credences: a mildly immodest proposal. Philosophy and Phenomenological Research, v. 92, n. 01, p. 1-24, 2016.

PETTIGREW, R. Epistemic Utility and Norms for Credences. Philosophy Compass, v. 8, n. 10, p. 897-908, 2013.

PETTIGREW, R. Accuracy and Evidence. Dialectica, v. 4, n. 67, p. 579-596, 2014a.

PETTIGREW, R. Accuracy, Risk, and the Principle of Indifference. Philosophy and Phenomenological Research, v. 92, n. 1, p. 35-59, 2014b.

PETTIGREW, R. Epistemic Utility Arguments for Probabilism. In: ZALTA, E. (Ed.). The Stanford Encyclopedia of Philosophy. Último Acesso em: 12 de dezembro de 2016, http://plato.stanford.edu/entries/epistemic-utility/, 2015.

PETTIGREW, R. Accuracy and the Laws of Credence. Oxford: Oxford University Press, 2016.

RAMSEY, F. P. Truth and Probability. In: RAMSEY, F. P.; BRAITHWAITE, R. B. (Eds.). The Foundations of Mathematics and Other Logical Essays. London: Routledge, 1950, p. 156-198.

ROSENKRANTZ, R. Foundations and Applications of Inductive Probability. Atascadero, CA: Ridgeview Press, 1981.

WEISBERG, J. Varieties of Bayesianism. In: GABBAY, D.; HARTMANN, S.; WOODS, J. (Eds.). Handbook of the History of Logic, vol. 10, Inductive Logic. Amsterdan: North-Holland, 2011, p. 477-551.

Recebido em: 10/10/2016

Aprovado para a publicação em: 13/02/2017

\begin{tabular}{|c|c|l|l|l|l|}
\hline intuitio & $\begin{array}{c}\text { ISSN } \\
1983-4012\end{array}$ & Porto Alegre & Vol.10 $-\mathrm{N}^{\circ} .1$ & $\begin{array}{l}\text { Julho } \\
2017\end{array}$ & p.15-24 \\
\hline
\end{tabular}

